\title{
Bone mineral density in vocational and professional ballet dancers
}

\author{
T. Amorim ${ }^{1,2} \cdot$ Y. Koutedakis ${ }^{2,3}$ - A. Nevill ${ }^{2}$ M. Wyon ${ }^{2,4}$ - J. Maia ${ }^{1}$ • \\ J. C. Machado ${ }^{5}$ - F. Marques ${ }^{6}$ - G. S. Metsios ${ }^{2,3}$ - A. D. Flouris ${ }^{3}$. \\ N. Adubeiro $^{7} \cdot$ L. Nogueira ${ }^{7} \cdot$ L. Dimitriou $^{8}$
}

Received: 15 September 2016 / Accepted: 14 June 2017 /Published online: 27 June 2017

(C) International Osteoporosis Foundation and National Osteoporosis Foundation 2017

\begin{abstract}
Summary According to existing literature, bone health in ballet dancers is controversial. We have verified that, compared to controls, young female and male vocational ballet dancers have lower bone mineral density (BMD) at both impact and non-impact sites, whereas female professional ballet dancers have lower BMD only at non-impact sites.

Introduction The aims of this study were to (a) assess bone mineral density (BMD) in vocational (VBD) and professional (PBD) ballet dancers and (b) investigate its association with body mass (BM), fat mass (FM), lean mass (LM), maturation and menarche.

Methods The total of 152 VBD ( $13 \pm 2.3$ years; 112 girls, 40 boys) and 96 controls ( $14 \pm 2.1$ years; 56 girls, 40 boys) and 184 PBD ( $28 \pm 8.5$ years; 129 females, 55 males $)$ and 160
\end{abstract}

T. Amorim

tania amorim@hotmail.com

1 Centre of Research, Education, Innovation and Intervention in Sport, Faculty of Sports, University of Porto, Rua Dr. Plácido Costa 91, 4200-450 Porto, Portugal

2 Faculty of Education, Health and Wellbeing, University of Wolverhampton, Walsall, UK

3 School of Sports and Exercise Sciences, University of Thessaly, Trikala, Greece

4 National Institute of Dance Medicine and Science, London, UK

5 i3S - Instituto de Investigação e Inovação em Saúde, Universidade do Porto, Porto, Portugal

6 Faculty of Pharmacy, University of Porto, Porto, Portugal

7 School of Health Technology of Porto, Polytechnic Institute of Porto, Porto, Portugal

8 London Sport Institute, Middlesex University, London, UK controls ( $27 \pm 9.5$ years; 110 female, 50 males) were assessed at the lumbar spine (LS), femoral neck (FN), forearm and total body by dual-energy X-ray absorptiometry. Maturation and menarche were assessed via questionnaires.

Results VBD revealed lower unadjusted BMD at all anatomical sites compared to controls $(p<0.001)$; following adjustments for Tanner stage and gynaecological age, female VBD showed similar BMD values at impact sites. However, no factors were found to explain the lower adjusted BMD values in VBD (female and male) at the forearm (non-impact site), nor for the lower adjusted BMD values in male VBD at the FN. Compared to controls, female PBD showed higher unadjusted and adjusted BMD for potential associated factors at the FN (impact site) $(p<0.001)$ and lower adjusted at the forearm $(p<0.001)$. Male PBD did not reveal lower BMD than controls at any site.

Conclusions Both females and males VBD have lower BMD at impact and non-impact sites compared to control, whereas this is only the case at non-impact site in female PBD. Maturation seems to explain the lower BMD at impact sites in female VBD.

Keywords Associated factors $\cdot$ Ballerinas $\cdot$ Bone mass $\cdot$ Elite dance $\cdot$ Prevalence

\section{Introduction}

Osteoporosis and osteopenia (i.e. low bone mineral density (BMD)) are recognised as the most frequent bone disorders, linked to high treatment costs and limited quality of life due to osteoporotic fractures [1, 2]. Hence, the identification of those at high risk is crucial for planning appropriate prevention programmes. The diagnosis of low BMD in premenopausal women and children is based on the International Society of Clinical Densitometry (ISCD) guideline, whereas a diagnosis 
is confirmed when BMD values lie within 2.0 standard deviation (SD) or more below the average value [3]. The American College of Sports Medicine (ACSM) has proposed different guidelines for the diagnosis in athletes. The term "low BMD" is used for BMD values between -1.0 and $-2.0 \mathrm{SD}$ and the term "osteoporotic" for BMD equal or less than $-2.0 \mathrm{SD}$ (along with secondary risk factors for stress fractures) [4].

Low BMD has been traditionally associated with the elderly and postmenopausal women [5], though some athletic populations, as endurance athletes, might also be at increased risk $[6,7]$. In ballet dancers, however, aspects regarding low BMD remain ambiguous [8]. While some authors underline the negative effects of professional dance training on bone metabolism (e.g. lean body type required for performance) [9-11], others suggest that the mechanical impact from dancing may provide a protection against low BMD, particularly at impact sites [12-14]. For instance, the high levels of muscular strength required for technical performance and weightbearing activity associated with jumping may stimulate bone-forming cells [12-14]. Nevertheless, most of the relevant publications on ballet dancers have been categorised average to low quality [8]. Therefore, the aims of the present study were (a) to assess BMD in vocational (VBD) and professional ballet dancers (PBD) and (b) to investigate the association between BMD with body mass (BM), fat mass (FM), lean mass (LM), menarche and maturation.

\section{Methods}

\section{Study population}

This study was conducted by inviting active students from vocational dance schools (children undergoing $4-8 \mathrm{~h}$ a day dance training in order to prepare for the profession) and active dancers from professional ballet companies. Pilot studies were administrated at a vocational dance school and a professional ballet company in order to calculate the sample size needed for prevalence estimate; sex- and age-matched controls were also included in both cases. In a sample of 36 female VBD and 36 matched controls, low BMD (Z-score of $<-2.0$ ) at the lumbar spine (LS) was found in $36 \%$ and $6 \%$, respectively. Based on this finding, we estimated that 37 participants were needed in each group to obtain $90 \%$ power, with $\alpha=0.05$. Similarly, in a sample of 22 female PBD (22 matched controls) and 10 male PBD (10 matched controls), the prevalence of low BMD (Z-score of -1.0 ) at the LS was found to be $32 \%$ (vs. 5\%) in female PBD and 20\% (vs. $0 \%$ ) in male PBD. We subsequently estimated that 42 female participants and 46 male participants in each group were needed to reach significance ( $90 \%$ power, $\alpha=0.05$ ). Assuming participants' non-response and possible dropouts, we approached two vocational dance schools and four professional ballet companies.

To recruit participants, an introductory letter briefly explaining the purposes of the study was initially forwarded to the executive boards of the dance schools and companies. Following the boards' agreement, the research team contacted the VBD (their guardians too) and PBD to present them with the study's aims and methodologies. From the total of 595 participants (360 VBD and 235 PBD), 158 VBD and 206 PBD volunteered. From this cohort, those who had received or were receiving medications known to affect bone metabolism were excluded (one PD), together with those receiving calcium supplements (two VD and one PD). Given the differences in bone mass values between individuals from different races [15], only participants referring to themselves as white European-Caucasian dancers were included. Based on these criteria, the total of 152 VBD ( $13 \pm 2.3$ years; 112 girls, 40 boys) and 184 PBD ( $28 \pm 8.5$ years; 129 females, 55 males $)$ were finally included in this study. Participants provided details on physical exercise (hours per week). Female and male VBD reported to perform $18.2 \pm 7.0$ and $19.5 \pm 7.2 \mathrm{~h}$ per week of dance training, respectively. Female and male PBD reported $32.9 \pm 8.4$ and $32.5 \pm 9.6 \mathrm{~h}$ per week of dance training, respectively. Details of the recruited dance population and its participation rate appear in Fig. 1.

Controls were also included in this study. Controls for the VBD were recruited from two local state schools, while controls for PBD were recruited from two local state universities. Eligibility criteria for controls were set according to dancers' characteristics, i.e. controls were only considered eligible if they were of the same sex, age (defined as decimal age; 12month difference of a dancer) and race (white EuropeanCaucasian). Exclusion criteria included current and previous participation in regular and organised physical activities. This rule did not apply to children participants involved in physical education sessions at their school. Control participation was also restricted to those who had received or were receiving medications known to affect bone metabolism. All participation criteria explaining the purpose for the recruitment were advertised via email and letters, following consent from the respective boards of directors. Out of the 282 responses (105 pupils, 177 university students), 256 fulfilled the current criteria and were included in the study (controls for VBD 96 (14 \pm 2.1 years), 56 girls, 40 boys; controls for PBD 160 (27 \pm 9.5 years), 110 female, 50 males). Female and male controls for VBD were involved in $2.4 \pm 0.5$ and $2.1 \pm 0.4 \mathrm{~h}$ per week of physical exercise, consisting mainly of school physical education. Female and male PBD controls did not report extra physical exercise apart from daily life routines. Details of the recruited controls and its participation rate appear in Fig. 2.

All participants provided signed informed consent. Following that, they underwent anthropometric measures, 


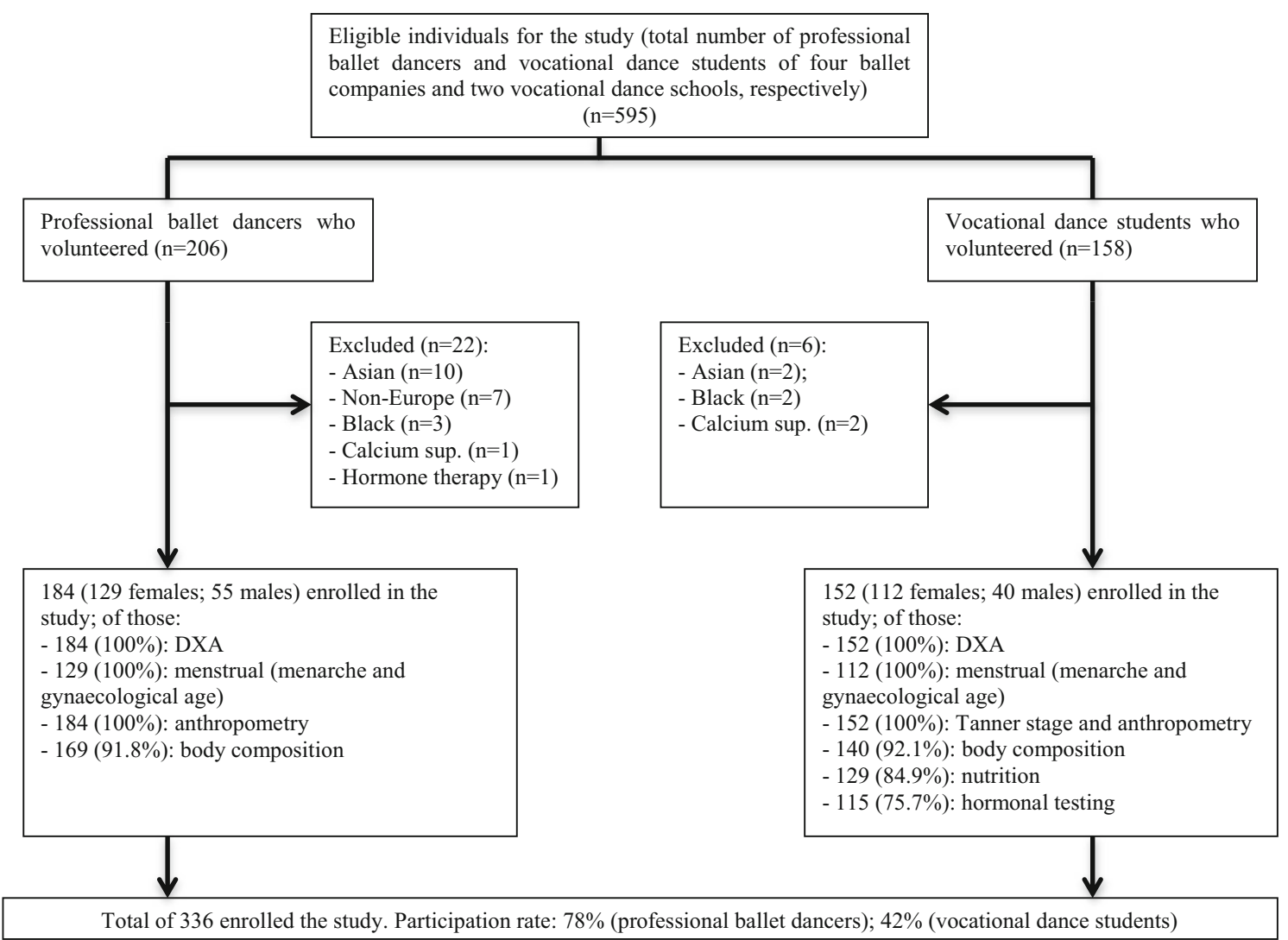

Fig. 1 Enrolment of dance population in the study

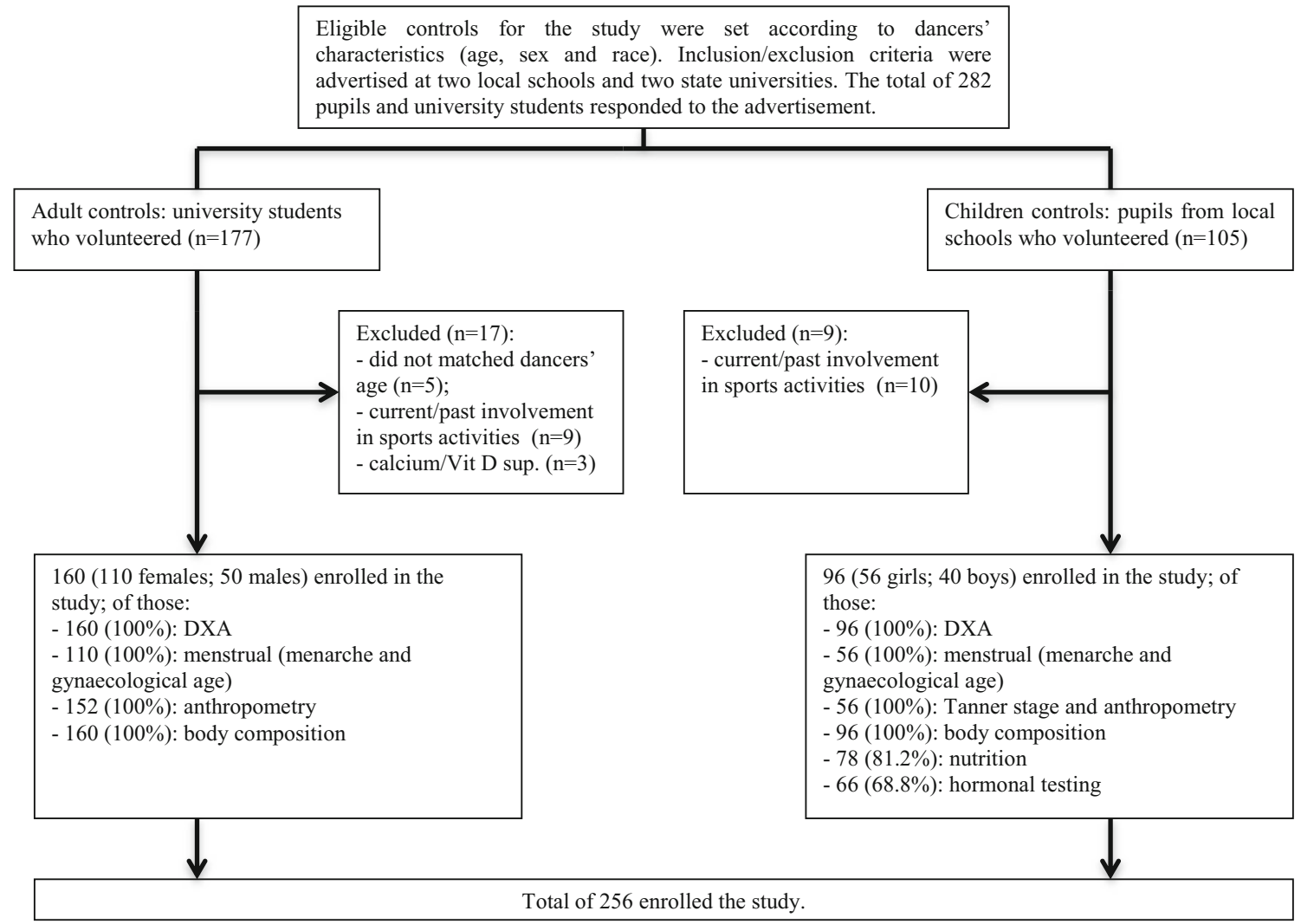

Fig. 2 Enrolment of control population in the study 
completed a menstrual questionnaire and participated in bone/ body composition measurements (Fig. 1). All procedures were approved by the NHS Health Research Authority, UK (Proc.14/WM/0008 and 14/WM/0009) and by the ethics committee of the Regional Administration of Health of Lisbon, Portugal (Proc.063/CES/INV/2012) in accordance with the Helsinki Declaration.

\section{Anthropometry measurements, menstruation, smoking, nutrition intake, hormonal analysis and pubertal assessment}

Chronological age was obtained as decimal age (date of birth minus measurement date). Participants' height (m), sitting height $(\mathrm{m})$ and BM $(\mathrm{kg})$ were measured using standard stadiometers (Seca) and digital scales (Tanita), respectively. BM index (BMI) was calculated as kilogrammes per square meter $\left(\mathrm{kg} \mathrm{m}^{-2}\right)$. Female participants completed a questionnaire to determine age at menarche. Total lifetime menses (number of menses since menarche to current age) were calculated as previously described [16]. Primary amenorrhea was defined as the absence of menarche by the age of 15 [17]. Gynaecological age (years) was calculated from the year of menarche to the age at which data were collected-current age [18].

Participants were asked to report their smoking history habits. Nutrient intakes were recorded via a validated 3-day food diary (two weekdays and one during weekend) [19]; this information was only assessed in VBD and their controls. The Food Processor SQL Edition version 9.8.1 was used to estimate average energy, calcium and vitamin D intakes.

Blood samples were collected in the early morning after an 8-h fasting. Serum insulin-like growth factor-1 (IGF-1) was measured by immunoradiometric assay kit (IRMA, IMMUNOTECH SAS, Marseille, France), in an automated analyser (Wallac Wizard 1470, Finland). The assay ranges were from 2 to $1.200 \mathrm{ng} \mathrm{mL}^{-1}$. The intra-assay and interassay CVs were below or equal to 6.3 and $6.8 \%$, respectively. Blood samples were centrifuged at $2500 \mathrm{~g}$ for $10 \mathrm{~min}$ and serum stored at $-80^{\circ} \mathrm{C}$ until analyses. Finally, pubertal development in VBD and their controls were self-reported using the Tanner sexual staging questionnaire [20].

\section{Body composition and bone measurements}

BMD at the LS, femoral neck (FN) and forearm (one-third distal radius) were measured using dual-energy X-ray absorptiometry (DXA). Body composition was assessed through a DXA whole-body scan (FM and LM (kg)). As participants were from different regions, two different DXA devices were used (Hologic (Discovery Wi) and Lunar (GE Lunar Prodigy)). The total of 68 (44.7\%) VBD and 178 (96.7\%) PBD were subjected to the Lunar scan device while the remaining 84 (55.3\%) VBD and 6 (3.3\%) PBD were scanned using Hologic. In addition, 20 (27.1\%) children controls and $110(68.8 \%)$ adult controls were assessed on a Lunar device vs. $70(72.9 \%)$ and $50(31.2 \%)$ on Hologic, respectively.

It is known that Lunar and Hologic BMD measurements demonstrate high correlation values between them [21, 22]. It is also known that there is a tendency for the Lunar model to inflate BMD values compared to the Hologic [22]. Therefore, besides the daily calibration required from each DXA manufacturer, cross-calibration of the two scanners was also conducted on a group of 20 men and women; the age of these 20 participants covered the age range of the entire sample (both dancers and controls) used for the purpose of the present study. The 20 participants were measured with both Lunar and Hologic within a period of 5 days. Subsequently, regression equations using BMD from Lunar as a dependent variable and BMD from Hologic as an independent variable were performed taking into account cross-calibration. The correlation between the two DXA models were high (forearm BMD $r=0.96$, adjusted $r^{2}=0.93$, std. error of estimate $=0.03$; LS BMD $r=0.96$, adjusted $r^{2}=0.92$, std. error of estimate $=0.05$; FN BMD $r=0.97$, adjusted $r^{2}=0.93$, std. error of estimate $=0.05$ ). The Hologic BMD data were further converted to the Lunar data using the following equations: forearm BMD Lunar $=-0.085263+1.356535 \times$ Hologic; LS BMD Lunar $=0.030762+1.161805 \times$ Hologic; FN BMD Lunar $=0.084782+1.116509 \times$ Hologic. Following the BMD adjustments, Z-scores at each anatomical site were further calculated for VBD considering standard data reference ranges for gender and age provided by the Lunar manufacturer (BMDCS data reference for children adjusted for height).

\section{Statistical analyses}

Independent t-tests were used to compare general characteristics between dance population and controls. Chi-square test was adopted to determine whether there is a significant difference in the distribution of Tanner stages between VBD and controls. Chi-square analyses were further employed to examine prevalence differences of low BMD between VBD (stratified by sex) and their controls. Analysis of covariance (ANCOVA) was conducted in VBD and PBD (also stratified by sex) in order to identify potential associated factors that might explain differences in BMD between groups (i.e. VBD $\times$ matched controls and PBD $\times$ matched controls). Consequently, BMD at each anatomical site (dependent variable) was adjusted for the following: BM, FM, LM, Tanner stage, age at menarche, gynaecological age and energy intake (covariates were entered as separate constituents). However, prior to the aforementioned analysis, all BMD data were controlled for school/company and/or DXA effect, since our dancers were (a) recruited from different ballet schools/ companies and (b) scanned using two DXA devices. 
Missing data were identified as "system missing" using the SPSS software-version 20.0. We had missing data for FM ( 7.9 and $8.2 \%$ in VBD and PBD, respectively) and nutrition intake (15.1 and $18.8 \%$ in VBD and controls, respectively). Statistical significance was set at $p<0.05$.

\section{Results}

Table 1 depicts the general characteristics of all participants. Table 1 indicates that maturity differences between dancers and controls are more pronounced in female VBD than those in their male counterparts. Compared to controls, female and male VBD revealed significantly lower BM (by 10.8 and $11.1 \mathrm{~kg}$, respectively; $p<0.001$ ), BMI (by 4.4 and $3.6 \mathrm{~kg} \mathrm{~m}^{-2}$, respectively; $p<0.001$ ) and FM (by 9.0 and $8.0 \mathrm{~kg}$, respectively; $p<0.001)$. In female VBD, age of menarche was $\sim 18$ months later than that in controls $(p<0.001)$. Similarly, female and male PBD revealed significantly lower BM (by 9.2 and $6.0 \mathrm{~kg}$, respectively; $p<0.001$ ) and BMI (by 3.9 and $2.0 \mathrm{~kg} \mathrm{~m}^{-2}$, respectively; $p<0.001$ ) compared to controls. Female PBD also demonstrated significantly lower FM (by $10.3 \mathrm{~kg}, p<0.001$ ) and higher LM (by $2 \mathrm{~kg}, p<0.01$ ) compared to controls and had their menarche approximately 2 years later than controls $(p<0.001)$. There was no significant difference between VBD and controls for calcium and vitamin $\mathrm{D}$ intakes, but both female and male VBD consumed significantly fewer calories per day compared to controls (by $215.1 \mathrm{kcal} /$ day or $13.2 \%$ and $278.0 \mathrm{kcal} /$ day or $17.4 \%$, respectively, $p<0.05)$. Serum IGF-1 concentrations were not significantly different in VBD compared to controls. Table 1 also depicts unadjusted BMD values for potential associated factors (i.e. BMD data were only adjusted for DXA device and school/company). Both female and male VBD show significantly lower unadjusted BMD values for potential associated factors at all measured anatomical sites compared to controls $(p<0.001)$. However, female PBD demonstrate significantly higher unadjusted BMD at the FN (by $11.9 \%, p<0.001$ ) and significantly lower unadjusted BMD at the forearm (by $13.9 \%, p<0.001)$. Male PBD show significantly higher unadjusted BMD values than controls at the FN (by $15.9 \%$, $p<0.001$ ) and LS (by $10.3 \%, p<0.01$ ).

Tables 2 and 3 depict the ANCOVA results for VBD and PBD, respectively. In particular, Table 2 illustrates that both female and male VBD have significantly lower adjusted BMD values at all anatomical sites compared to controls. BM, LM, FM and energy intake were positively associated with BMD in female VBD at the FN $(p<0.001, p<0.001, p<0.01$ and $p<0.05$, respectively). However, these covariates did not explain group differences (i.e. VBD vs. controls); only when controlling for Tanner stage and gynaecological age, BMD differences between groups were dissipated. The factors determining BMD differences between VBD and their matched controls at the LS were Tanner stage (females and males both at $p<0.001$ ) and body mass (only for males, $p<0.001$ ). No factors were detected to explain the lower adjusted BMD values in VBD (both in female and male) at the forearm (non-impact site) than those of controls, nor for the lower adjusted BMD values in male VBD at the FN (impact site).

Table 3 confirms that our female PBD have higher adjusted BMD values at the FN $(p<0.001)$ and lower adjusted BMD values at the forearm $(p<0.001)$ than those for the controls. LM and gynaecological age were positively associated with these findings at the $\mathrm{FN}$ ( $p<0.05, p<0.001$, respectively); the fact that our female PBD had their menarche later than the controls seems to explain the BMD differences between groups at the forearm $(p<0.001)$. FM is positively associated with BMD at the LS in female PBD $(p<0.01)$. Male PBD revealed higher adjusted $\mathrm{BMD}$ at impact sites than controls (FN and LS) and similar BMD values at the forearm; LM is positively associated with these findings at the $\operatorname{LS}(p<0.01)$.

Table 4 shows the prevalence of low BMD in VBD (Zscore $<-2.0)$. Significantly higher prevalence of low BMD at the forearm (9.2 vs. $0 \%, p=0.01)$ and LS (16.4 vs. $5.5 \%$, $p<0.05$ ) was noted in the female VBD compared to that in the controls. Although not significant, the proportion of cases with low BMD was higher in male VBD at all anatomical sites compared to that in the controls.

\section{Discussion}

Data on BMD in dancers has been ambiguous thus far. This is supported by a recent systematic review highlighting the need for further research on the field [8]. To our knowledge, the present study is the first to compare BMD values in a relatively large cohort of both vocational and professional ballet dancers. We found that female and male VBD have lower BMD values compared to matched controls at both impact (FN and LS) and non-impact (forearm) sites. It is noteworthy that the proportion of cases with low BMD $(Z$-score $<-2.0)$ in female VBD was significantly higher compared to that in controls at both impact (LS) and non-impact (forearm) sites; although not significant, male VBD demonstrated higher prevalence of low BMD at all three assessed anatomical sites. Nevertheless, after adjusting BMD for maturation markers (Tanner stage and gynaecological age), we found similar values at impact sites (both FN and LS) in female VBD. This means that BMD differences between groups at these sites can be explained by the fact that our female VBD dancers are late matures compared to controls. However, maturation markers did not explain the lower BMD displayed by VBD (both female and male) at non-impact sites compared to that by controls, nor the lower BMD in male VBD at the LS. Considering female PBD, we found significantly higher unadjusted and adjusted BMD values at impact sites (FN) and 


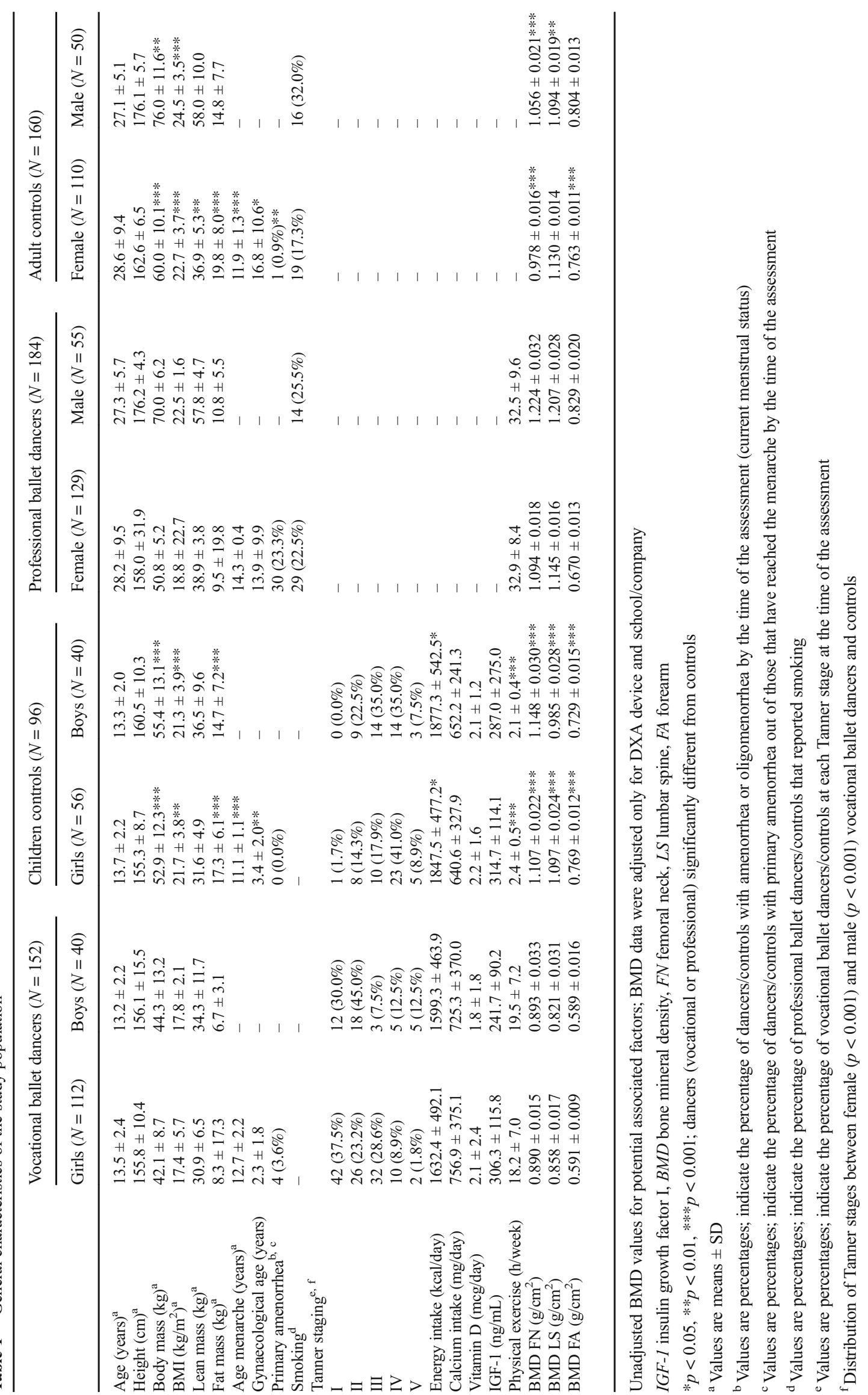


Table 2 Factors associated with BMD in vocational ballet dancers (children)

\begin{tabular}{|c|c|c|c|c|c|c|c|c|}
\hline & \multicolumn{2}{|l|}{ Female } & \multirow[t]{2}{*}{$\mathrm{F}$} & \multirow[t]{2}{*}{$\mathrm{P}$} & \multicolumn{2}{|l|}{ Male } & \multirow[t]{2}{*}{$\mathrm{F}$} & \multirow[t]{2}{*}{$\mathrm{P}$} \\
\hline & Dancers & Controls & & & Dancers & Controls & & \\
\hline \multicolumn{9}{|l|}{ BMD femoral neck ${ }^{\mathrm{a}}$} \\
\hline Dancer vs. control & $0.591 \pm 0.009$ & $0.768 \pm 0.012 * * *$ & 129.863 & 0.000 & $0.589 \pm 0.016$ & $0.729 \pm 0.015 * * *$ & 39.095 & 0.000 \\
\hline Body mass & $0.943 \pm 0.013$ & $1.034 \pm 0.024 * *$ & 97.566 & 0.000 & $0.968 \pm 0.025$ & $1.077 \pm 0.026^{* *}$ & 56.224 & 0.000 \\
\hline Lean mass & $0.593 \pm 0.008$ & $0.770 \pm 0.011 * * *$ & 68.840 & 0.000 & $0.590 \pm 0.011$ & $0.727 \pm 0.010 * * *$ & 94.281 & 0.000 \\
\hline Fat mass & $0.613 \pm 0.010$ & $0.730 \pm 0.016^{* * *}$ & 12.334 & 0.001 & $0.588 \pm 0.018$ & $0.729 \pm 0.017 * * *$ & 0.005 & 0.946 \\
\hline Tanner stage & $0.956 \pm 0.013$ & $0.968 \pm 0.026$ & 107.406 & 0.000 & $0.987 \pm 0.025$ & $1.073 \pm 0.025^{*}$ & 70.829 & 0.000 \\
\hline Age at menarche & $0.991 \pm 0.026$ & $1.151 \pm 0.031 * * *$ & 1.379 & 0.243 & - & - & - & - \\
\hline Gynaecological age & $1.025 \pm 0.023$ & $1.098 \pm 0.028$ & 14.879 & 0.000 & - & - & - & - \\
\hline Energy intake & $0.897 \pm 0.015$ & $1.179 \pm 0.052 * * *$ & 6.144 & 0.014 & $0.901 \pm 0.032$ & $1.084 \pm 0.032 * * *$ & 5.524 & 0.022 \\
\hline \multicolumn{9}{|l|}{ BMD lumbar spine ${ }^{a}$} \\
\hline Dancer vs. control & $0.858 \pm 0.017$ & $1.097 \pm 0.024 * * *$ & 65.568 & 0.000 & $0.821 \pm 0.031$ & $0.985 \pm 0.028 * * *$ & 15.120 & 0.000 \\
\hline Body mass & $0.917 \pm 0.013$ & $1.016 \pm 0.025^{* *}$ & 136.834 & 0.000 & $0.890 \pm 0.021$ & $0.907 \pm 0.021$ & 107.557 & 0.000 \\
\hline Lean mass & $0.866 \pm 0.014$ & $1.095 \pm 0.020 * * *$ & 91.746 & 0.000 & $0.824 \pm 0.019$ & $0.978 \pm 0.017 * * *$ & 145.894 & 0.000 \\
\hline Fat mass & $0.902 \pm 0.019$ & $1.023 \pm 0.030^{* *}$ & 11.139 & 0.001 & $0.828 \pm 0.035$ & $0.975 \pm 0.032 * *$ & 0.220 & 0.640 \\
\hline Tanner stage & $0.927 \pm 0.014$ & $0.954 \pm 0.028$ & 117.896 & 0.000 & $0.906 \pm 0.022$ & $0.900 \pm 0.021$ & 100.105 & 0.000 \\
\hline Age at menarche & $0.972 \pm 0.027$ & $1.159 \pm 0.032 * * *$ & 0.514 & 0.476 & - & - & - & - \\
\hline Gynaecological age & $1.007 \pm 0.024$ & $1.103 \pm 0.029^{*}$ & 19.896 & 0.000 & - & - & - & - \\
\hline Energy intake & $0.866 \pm 0.017$ & $1.074 \pm 0.060 * *$ & 1.317 & 0.253 & $0.823 \pm 0.030$ & $0.911 \pm 0.031 *$ & 6.591 & 0.013 \\
\hline \multicolumn{9}{|l|}{ BMD forearm ${ }^{\mathrm{a}}$} \\
\hline Dancer vs. control & $0.591 \pm 0.009$ & $0.768 \pm 0.012 * * *$ & 129.863 & 0.000 & $0.589 \pm 0.016$ & $0.729 \pm 0.016^{* * *}$ & 39.095 & 0.000 \\
\hline Body mass & $0.617 \pm 0.007$ & $0.739 \pm 0.014 * * *$ & 86.416 & 0.000 & $0.617 \pm 0.012$ & $0.692 \pm 0.013 * * *$ & 68.208 & 0.000 \\
\hline Lean mass & $0.593 \pm 0.008$ & $0.770 \pm 0.011 * * *$ & 69.840 & 0.000 & $0.590 \pm 0.011$ & $0.727 \pm 0.010 * * *$ & 94.281 & 0.000 \\
\hline Fat mass & $0.613 \pm 0.010$ & $0.730 \pm 0.016^{* * *}$ & 12.334 & 0.001 & $0.588 \pm 0.018$ & $0.729 \pm 0.017 * * *$ & 0.005 & 0.946 \\
\hline Tanner stage & $0.632 \pm 0.070$ & $0.690 \pm 0.014 * * *$ & 137.451 & 0.000 & $0.613 \pm 0.011$ & $0.686 \pm 0.011^{* *}$ & 113.363 & 0.000 \\
\hline Age at menarche & $0.644 \pm 0.015$ & $0.802 \pm 0.017 * * *$ & 0.003 & 0.960 & - & - & - & - \\
\hline Gynaecological age & $0.656 \pm 0.014$ & $0.782 \pm 0.016^{* * *}$ & 11.336 & 0.001 & - & - & - & - \\
\hline Energy intake & $0.597 \pm 0.009$ & $0.781 \pm 0.030 * * *$ & 1.272 & 0.262 & $0.582 \pm 0.017$ & $0.692 \pm 0.017 * * *$ & 4.560 & 0.037 \\
\hline
\end{tabular}

Adjusted BMD values for potential associated factors; BMD data were adjusted also for DXA-device and school/company

$B M D$ bone mineral density

$* p<0.05, * * p<0.01, * * * p<0.001$; dancers (vocational or professional) significantly different from controls

${ }^{\mathrm{a}}$ Values are means $\pm \mathrm{SD}$

significantly lower BMD at the forearm compared to that of matched controls. These findings suggest that weight-bearing exercise might be able to improve BMD despite a relatively low BM, an indication that such exercise might be able to override any potential negative effect. A similar result has been obtained for male PBD who did not reveal lower BMD compared to controls at any site. The latter confirms previous data [23] and could be partly explained by the fact that males have less pronounced endocortical resorption and higher periosteal expansion compared to females [24].

Dancing has been considered as a weight-bearing activity [13]. Studies using weight-bearing physical activities have shown positive effects on bone mineral accrual in both adults and children $[25,26]$. Indeed, it has been suggested that $60 \mathrm{~min} \times 3 \mathrm{a}$ week of weight-bearing exercise is sufficient to prevent low BMD in the general population [27]. Since our participants were vocational and professional dancers, they were involved in daily classes of several hours of weightbearing activity $[28,29]$. Considering data on bone cell biology and function of osteocytes as mechanosensory cells [30, 31], it would be expected to find significantly higher BMD values at impact sites (particular FN) and similar BMD values at non-impact sites compared to those of controls. However, dancing is also an aesthetic activity whereas body size is essential for performance. This requirement might place dancers at risk for low BM, a well-known risk factor for low bone mass phenotypes. Indeed, in our study, both VBD and PBD had significantly lower BM values compared to their controls. Further, compared to matched controls, female PBD also revealed higher prevalence of primary amenorrhea (and latter 
Table 3 Factors associated with BMD in professional ballet dancers (adults)

\begin{tabular}{|c|c|c|c|c|c|c|c|c|}
\hline & \multicolumn{2}{|l|}{ Female } & \multirow[t]{2}{*}{$\mathrm{F}$} & \multirow[t]{2}{*}{$\mathrm{P}$} & \multicolumn{2}{|l|}{ Male } & \multirow[t]{2}{*}{$\mathrm{F}$} & \multirow[t]{2}{*}{$\mathrm{P}$} \\
\hline & Dancers & Controls & & & Dancers & Controls & & \\
\hline \multicolumn{9}{|l|}{ BMD femoral neck ${ }^{\mathrm{a}}$} \\
\hline Dancer vs. control & $1.094 \pm 0.018$ & $0.978 \pm 0.016 * * *$ & 22.067 & 0.000 & $1.224 \pm 0.032$ & $1.056 \pm 0.021 * * *$ & 19.556 & 0.000 \\
\hline Body mass & $1.099 \pm 0.033$ & $0.977 \pm 0.016^{* *}$ & 3.325 & 0.070 & $1.224 \pm 0.033$ & $1.056 \pm 0.021 * * *$ & 0.000 & 0.990 \\
\hline Lean mass & $1.093 \pm 0.019$ & $0.918 \pm 0.016^{* * *}$ & 4.179 & 0.042 & $1.272 \pm 0.038$ & $1.053 \pm 0.021 * * *$ & 0.789 & 0.378 \\
\hline Fat mass & $1.111 \pm 0.021$ & $0.967 \pm 0.018 * * *$ & 0.931 & 0.336 & $1.261 \pm 0.039$ & $1.057 \pm 0.021 * * *$ & 1.587 & 0.213 \\
\hline Age at menarche & $1.096 \pm 0.035$ & $0.981 \pm 0.017$ & 0.511 & 0.476 & - & - & - & - \\
\hline Gynaecological age & $1.088 \pm 0.032$ & $0.987 \pm 0.015^{* *}$ & 18.386 & 0.000 & - & - & - & - \\
\hline \multicolumn{9}{|l|}{ BMD lumbar spine ${ }^{a}$} \\
\hline Dancer vs. control & $1.145 \pm 0.016$ & $1.113 \pm 0.014$ & 2.106 & 0.148 & $1.207 \pm 0.028$ & $1.094 \pm 0.019 * *$ & 10.845 & 0.002 \\
\hline Body mass & $1.122 \pm 0.029$ & $1.096 \pm 0.014$ & 11.904 & 0.001 & $1.224 \pm 0.028$ & $1.086 \pm 0.019$ & 7.472 & 0.008 \\
\hline Lean mass & $1.153 \pm 0.017$ & $1.117 \pm 0.014$ & 2.719 & 0.101 & $1.240 \pm 0.032$ & $1.098 \pm 0.019 * * *$ & 8.128 & 0.006 \\
\hline Fat mass & $1.194 \pm 0.019$ & $1.090 \pm 0.015 * * *$ & 12.370 & 0.001 & $1.260 \pm 0.037$ & $1.099 \pm 0.020 * * *$ & 0.172 & 0.679 \\
\hline Age at menarche & $1.123 \pm 0.030$ & $1.103 \pm 0.014$ & 5.547 & 0.018 & - & - & - & - \\
\hline Gynaecological age & $1.102 \pm 0.029$ & $1.116 \pm 0.013$ & 6.186 & 0.014 & - & - & - & - \\
\hline \multicolumn{9}{|l|}{ BMD forearm ${ }^{\mathrm{a}}$} \\
\hline Dancer vs. control & $0.670 \pm 0.013$ & $0.763 \pm 0.011 * * *$ & 27.337 & 0.000 & $0.829 \pm 0.020$ & $0.804 \pm 0.013$ & 1.228 & 0.272 \\
\hline Body mass & $0.587 \pm 0.024$ & $0.754 \pm 0.012 * *$ & 1.370 & 0.243 & $0.837 \pm 0.020$ & $0.800 \pm 0.013$ & 3.739 & 0.057 \\
\hline Lean mass & $0.671 \pm 0.014$ & $0.766 \pm 0.012 * * *$ & 0.535 & 0.465 & $0.848 \pm 0.025$ & $0.806 \pm 0.014$ & 0.243 & 0.624 \\
\hline Fat mass & $0.685 \pm 0.015$ & $0.755 \pm 0.013 * *$ & 4.990 & 0.027 & $0.858 \pm 0.024$ & $0.803 \pm 0.013$ & 4.553 & 0.037 \\
\hline Age at menarche & $0.708 \pm 0.024$ & $0.742 \pm 0.012$ & 14.861 & 0.000 & - & - & - & - \\
\hline Gynaecological age & $0.683 \pm 0.022$ & $0.758 \pm 0.010 * *$ & 24.885 & 0.000 & - & - & - & - \\
\hline
\end{tabular}

Adjusted BMD values for potential associated factors; BMD data were adjusted also for DXA-device and school/company

$B M D$ bone mineral density

$* p<0.05, * * p<0.01, * * * p<0.001$; dancers (vocational or professional) significantly different from controls

${ }^{\text {a }}$ Values are means $\pm \mathrm{SD}$

age at menarche), another well-known osteoporosis risk factor. Nevertheless, the fact that female PBD showed higher BMD at impact sites compared to controls suggests that dance training is able to stimulate BMD gains, even in the presence of osteoporosis risk factors. Indeed, female PBD only revealed lower BMD values compared to non-exercising controls at the forearm (non-impact site), which might indicate that exercise (dance training) can counterbalance the potential negative effects of osteoporosis risk factors at loading sites. However, it seems such a compensatory effect could not be seen in VBD since they demonstrated significantly lower bone mass at all studied anatomical sites. Actually, the prevalence of low

Table 4 Prevalence of low bone mineral density in vocational dancers and age- and sex-matched controls

ISCD criteria

\begin{tabular}{|c|c|c|c|c|c|c|}
\hline & \multicolumn{2}{|l|}{ LS $(\%)$} & \multicolumn{2}{|l|}{$\mathrm{FN}(\%)$} & \multicolumn{2}{|l|}{ FA $(\%)$} \\
\hline & Female & Male & Female & Male & Female & Male \\
\hline Vocational dancers & 16.4 & 7.5 & 8.3 & 10.5 & 9.2 & 2.5 \\
\hline Controls & $5.5^{*}$ & 2.1 & 12.7 & 4.2 & $0.0^{* *}$ & 2.2 \\
\hline
\end{tabular}

Values are percentages. The International Society of Clinical Densitometry (ISCD) criterion was used to assess vocational dancers and age- and sexmatched controls: "low BMD" was defined for a Z-score less than -2.0

$L S$ lumbar spine, $F N$ femoral neck, $F A$ forearm

$* p<0.05, * * p<0.01, * * * p<0.001$; dancers significantly different from controls (chi-square test) 
BMD at the forearm and LS was also significantly higher in female VBD compared to that in controls. As LS is mainly constituted by the trabecular bone (known to be more sensitive to mechanical stress from exercise [32]), and as ballet dancing requires high levels of muscular strength (placing considerable mechanical stress on the lower back [28, 33]), it would not be expected to find a significantly higher number of cases with low BMD at this anatomical site compared to controls. It seems logical to suggest maturation as the reason for these findings in female VBD. Indeed, a disproportionally high number of VBD were at Tanner stage I compared to controls, which might indicate that dancers are late matures. Delayed puberty has been linked with low BMD in children and adolescents [34]. Further, maturation markers (i.e. Tanner stage and gynaecological age) seem also to explain the differences in BMD at the FN in female VBD. This finding is not surprising due to selection criteria for professional dance training; children have to go through an audience for a place in a vocational dance school, where specific body stereotypes (small body size; ecto-mesomorphic body type) are essential for acceptance [35]. However, although maturation seems to explain the group differences in BMD at impact sites, this is not the case when the forearm (non-impact site) is considered. Indeed, in line with available data [10, 18, 36-39], age at menarche, together with BM, LM, FM and energy intake, was significantly associated with BMD at the forearm; nevertheless, these factors do not seem to explain BMD differences between female VBD and controls at this anatomical site. Considering male VBD, the present study did not find factors to explain the lower BMD values compared to controls at both impact (FN) and non-impact sites. Previous studies usually focus on female dancers as it is generally accepted that females have increased odds for low BMD. However, the present study suggests that young male dancers may also be at risk for low BMD. Future studies should also consider young male dancers in relation to BMD in different settings. Further, factors such as low energy availability, genetics and/or hormonal levels should be considered in future studies, given their association with low bone mass phenotypes [4, 40].

The current results regarding BMD in VBD might be of concern, as young dancers may enter adulthood with relatively low BMD, which may further impair the peak bone mass attainment [41]. Delayed puberty has been reported to be associated with lower IGF-1 levels and low bone mass in children and adolescents [34]; interestingly though, serum IGF-1 was not significantly different between VBD and controls (both in female and male), despite the difference seen in Tanner staging. Nevertheless, findings in children should be interpreted with caution due to biological changes which occur during growth [41]. Longitudinal studies should be conducted in VBD to ascertain how bone mass changes throughout growing.
The clinical significance of low BMD lies on the increased risk of fracture [3, 4]. We did not record fractures or injuries among our studied population. Nevertheless, recent data have shown that over a 1 -year period the incidence of injury in VBD was 1.42 per student and the risk of injury $76 \%$ [42]. Also, in PBD, a total of 355 injuries were recorded during a year, with an overall incidence of 6.8 injuries per dancer [43]. However, to our knowledge, there are no available data on the association between dance injuries and low BMD [8]. Notwithstanding, the prevalence of $\mathrm{Z}$-scores below -1.0 is significantly higher among our dance population compared with that among controls. Indeed, since athletes in weight-bearing sports usually have 5-15\% higher BMD than non-athletes [4], the ACSM emphasises that a BMD Z-score of $<-1.0$ in athletic populations should be further investigated, even in the absence of fractures [4]. However, to the best of our knowledge, there are no preventative/screening measures in the dance population regarding overall dancers' bone health yet.

It is reasonable to assume that the present study might have been influenced by methodological limitations such as the use of a self-reported questionnaire to assess age at menarche, gynaecological age and Tanner stage. We also acknowledge the lack of injury and fracture records for our participants as well as alcohol intake. Another limitation may be that the current data incorporate dancers born and raised in north or south Europe, but performing at the same company. We further recognise the potential selection bias of the current participants since they were recruited from specific geographic regions. Finally, the assessment of bone geometry, a known determinant of bone strength, should also be considered in future studies to further substantiate the findings of this study.

\section{Conclusions}

Compared to controls, female and male vocational ballet dancers demonstrated lower bone mineral density at impact and non-impact sites; maturation markers in the young female vocational dancers seem to explain these findings only at impact sites. In contrast, unlike male professional dancers who demonstrated a healthy bone mineral density profile, their female counterparts revealed lower bone mass at the studied non-impact site compared to controls, but higher values at impact sites. Future studies should explore how bone mass changes as vocational dancers grow and progress to professional level.

Acknowledgements This work was supported by the Portuguese Foundation for Science and Technology [PhD grant number SFRH/BD/ $88585 / 2012]$. Thanks are expressed to the radiology services of the 
Beatriz Angelo Hospital. A very special thanks goes to all participants who volunteered.

\section{Compliance with ethical standards}

Conflicts of interest None.

\section{References}

1. Harvey N, Dennison E, Cooper C (2010) Osteoporosis: impact on health and economics. Nat Rev Rheumatol 6(2):99-105

2. Barrett-Connor E (1995) The economic and human costs of osteoporotic fracture. Am J Med 98(suppl2A):S3-S8

3. International Society for Clinical Densitometry. Updated 2013 official positions for adult and pediatric. http://www.iscd.org/ documents/2014/02/2013-iscd-official-position-brochure.pdf (Accessed May 2, 2016).

4. Nattiv A, Loucks AB, Manore MM et al (2007) American College of Sports Medicine position stand: the female athlete triad. Med Sci Sports Exerc 39(10):1867-1882

5. World Health Organization (2004) WHO scientific group on the assessment of osteoporosis at primary health care level. WHO Summary Meeting Report. WHO, Brussels

6. Scofield KL, Hecht S (2012) Bone health in endurance athletes: runners, cyclists, and swimmers. Curr Sports Med Rep 11(6): 328-334

7. Pollock N, Grogan C, Perry M, Pedlar C, Cooke K, Morrissey D et al (2010) Bone mineral density and other features of the female athlete triad in elite endurance runners. Int J Sport Nutr Exerc Metab 20:418-426

8. Amorim T, Wyon M, Maia J et al (2015) Prevalence of low bone mineral density in female dancers. Sports Med 45:257-268

9. Burckhardt P, Wynn E, Krieg MA et al (2011) The effects of nutrition, puberty and dancing on bone density in adolescent ballet dancers. J Dance Med 15(2):51-60

10. Keay N, Fogelman I, Blake G (1997) Bone mineral density in professional female dancers. Br J Sports Med 31:143-147

11. Dolyle-Lucas AF, Akers JD, Davy BM (2010) Energetic efficiency, menstrual irregularity, and bone mineral density in elite professional female ballet dancers. J Dance Med Sci 14(4):146-154

12. Litchtenbelt WD, Fogelholm M, Otteenheijm R et al (1995) Physical activity, body composition and bone density in ballet dancers. Br J Nutr 74:439-451

13. Khan KM, Green RM, Saul A et al (1996) Retired elite female ballet dancers and nonathletic controls have similar bone mineral density at weightbearing sites. J Bone Miner Res 11(10):15661574

14. To W, Wong M (2011) Does oligomenorrhea/amenorrhea and underweight imply athlete female trial syndrome in young female dancers? Eur J Sport Sci 11(5):335-340

15. Bachrach LK, Hastie T, Wang MC et al (1999) Bone mineral acquisition in healthy Asian, Hispanic, black, and Caucasian youth: a longitudinal study. J Clin Endocrinol Metab 84:4702-4712

16. Cobb KL, Bachrach LK, Greendale G et al (2003) Disordered eating, menstrual irregularity and bone mineral density in female runners. Med Sci Sports Exerc 35:711-719

17. Practice Committee of the American Society for Reproductive Medicine (2004) Current evaluation of amenorrhea. Fertil Steril $82: 266-272$

18. Dimitriou L, Weiler R, Lloyd-Smith R, Turner A, Heath L, Nic J, Reid A (2014) Bone mineral density, rib pain and other features of the female athlete triad in elite light weight rowers. BMJ Open 4(2): $1-9$
19. Crawford PB, Obarzaner E, Morrison J, Sabry ZI (1994) Comparative advantage of 3-day food records over 24-hour recall and 5-day food frequency validated by observation of 9- and 10year-old girls. J Am Diet Assoc 94(6):626-630

20. Duke PM, Litt IG, Gross RT (1980) Adolescent' self-assessment of sexual maturation. Pediatrics 66(6):918-920

21. Pocock NA, Sambrook PN, Nguyen T et al (1992) Assessment of spinal and femoral bone density by dual X-ray absorptiometry: comparison of lunar and hologic instruments. J Bone Miner Res 7(9):1081-1084

22. Hagiwara S, Engelke K, Yang S et al (1994) Dual X-ray absorptiometry forearm software: accuracy and intermachine relationship. J Bone Miner Res 9(9):1425-1427

23. Fredericson M, Chew K, Ngo J, Cleek T, Kiratli J, Cobb K (2007) Regional bone mineral density in male athletes: a comparison of soccer players, runners and controls. Br J Sports Med 41(10):664 668

24. Holroyd C, Cooper C, Dennison E (2008) Epidemiology of osteoporosis. Best Pract Res Clin Endocrinol Metab 22(5):671-685

25. Guadalupe-Grau A, Fuentes T, Guerra B et al (2009) Exercise and bone mass in adults. Sports Med 39(6):439-468

26. Greene DA, Naughton GA (2006) Adaptive skeletal responses to mechanical loading during adolescence. Sports Med 36(9):723-732

27. Vainionpää A, Korpelainen R, Leppäluoto J, Jämsä T (2005) Effects of high-impact exercise on bone mineral density: a randomized controlled trial in premenopausal women. Osteoporosis Int 16(2):191-197

28. Koutedakis Y, Sharp NC (2004) Thigh-muscles strength training, dance exercise, dynamometry, and anthropometry in professional ballerinas. J Strength Cond Res 18(4):714-718

29. Twitchett T, Angioi M, Koutedakis Y et al (2009) Video analysis of classical ballet performance. J Dance Med Sci 13(4):124-128

30. Bonewald LF (2011) The amazing osteocyte. J Bone Miner Res 26(2):229-238

31. Bonewald LF, Johnson ML (2008) Osteocytes, mechanosensing and Wnt signaling. Bone 42:606-615

32. Heinonen A, Sievänen H, Kannus P, Oja P, Pasanen M, Vuori I (2000) High-impact exercise and bones of growing girls: a 9month controlled trial. Osteoporosis Int 11:1010-1017

33. Koutedakis Y, Jamurtas AZ (2004) The dancer as a performing athlete: physiological considerations. Sports Med 34(10):651-661

34. Bounjour J, Chevalley T (2014) Pubertal timing, bone acquisition, and risk of fracture throughout life. Endocr Rev 35(5):820-847

35. Claessens ALM, Beunen GP, Nuyts MM et al (1987) Body structure, somatotype, maturation and motor performance of girls in ballet schooling. J. Sports Med 27:310-317

36. Peel N (2014) Disorders of bone metabolism. Surgery 33(1):15-20

37. Ma N, Gordon C (2012) Pediatric osteoporosis: where are we now. J Pediatr 161(6):983-990

38. Hage RPE, Courteix D, Benhamou CL, Jacob C, Jaffré C (2009) Relative importance of lean and fat mass on bone mineral density in a group of adolescent girls and boys. Eur J Appl Physiol 105(5): 759-764

39. Ilich JZ, Kerstetter JE (2000) Nutrition in bone health revisited: a story beyond calcium. J Am Coll Nutr 19(6):715-737

40. Eisman JA (1999) Genetics of osteoporosis. Endocr Rev 20(6): 788-804

41. Heaney RP, Abrams S, Dawson-Hughes B et al (2000) Peak bone mass. Osteoporosis Int 11:985-1009

42. Ekegren CL, Quested R, Brodrick A (2014) Injuries in preprofessional ballet dancers: incidence, characteristics and consequences. J Sci Med Sport 17(3):271-275

43. Allen N, Nevill AM, Brooks JH et al (2012) Ballet injuries: injury incidence and severity over 1 year. J Orthop Sports Phys Ther 42(9):780-790 